\title{
DILATIONS ON INVERTIBLE SPACES $\left({ }^{1}\right)$
}

\author{
BY \\ ELLARD NUNNALLY
}

\begin{abstract}
This paper primarily concerns certain groups of homeomorphisms which are associated in a natural way with a variety of spaces, which satisfy a set of axiomatic conditions put forth in $\S 1$.

Let us suppose that $X$ is a space of the type in question and that $G$ is an appropriate group of homeomorphisms of $X$ onto itself. In $\$ 2$ we demonstrate the existence of a nonvoid subcollection $\mathscr{D}$, the "topological dilations," of $G$ which is characterized in Theorem 1 in the following fashion: suppose $f \in \mathscr{D}$ and $g \in G$, then $g \in \mathscr{D}$ if and only if $f$ is a $G$-conjugate of $g$, that is if and only if there exists an element $h$ of $G$ such that $f=h g h^{-1}$.

We proceed then to show in $\$ 3$ that if $f$ and $g$ are nonidentity elements of $G$, then we may find $\delta, r \in G$ such that the product $\left(r g r^{-1}\right)\left(\delta f \delta^{-1}\right) \in \mathscr{D}$. We then combine this fact with the characterization of $\mathscr{D}$ mentioned above to conclude that each element of $\mathscr{D}$ is a "universal" element of $G$ in the sense that if $d \in \mathscr{D}$, then any element $g$ of $G$ may be represented as the product of two $G$-conjugates of $d$. Furthermore we conclude that if $g$ is not the identity element of $G$, then $g$ can be represented as the product of three $G$-conjugates of any nonidentity element of $G$.

Finally, we apply the conclusions to groups of homeomorphisms of certain spaces: for example spheres, cells, the Cantor set, etc.
\end{abstract}

1. Definitions, notation, and axioms. If $X$ is a topological space, $A$ and $B$ are subsets of $X$, and $f$ is a mapping of $X$ to itself, then we write ' $A \subseteq B^{\prime}$ for ' $A$ is a subset of $B^{\prime}$, and ' $A \subset B^{\prime}$ for ' $A \subseteq B$ and $A \neq B^{\prime}$. The mapping $f$ is said to be supported on $A$ if $f(x)=x$ whenever $x \in \sim A$. $A$ will be called a perfect subset of $X$ if $A \neq \varnothing$ and no open subset of $X$ contains exactly one point of $A$. Throughout this paper all mappings under consideration will be homeomorphisms of some space $X$ onto itself.

Henceforth we suppose that we have given:

(a) $X$ : a regular, first countable, Hausdorff space.

(b) $X^{\prime}:$ a perfect subspace of $X$.

(c) $(\mathscr{U}, \mathscr{K}): \mathscr{U}$ is a collection of open subsets of $X$ each having a nonvoid

Presented to the Society, November 15, 1963 under the title Stable homeomophisms of $S_{r}$; received by the editiors March 25, 1965.

(1) This constitutes the author's dissertation which was directed by Professor R. D. Anderson whom the author wishes to thank for his great assistance throughout the years. 
intersection with $X^{\prime}$, and each point of $X^{\prime}$ has a neighborhood basis contained in $\mathscr{U} . \mathscr{K}$ will denote the collection of the closures of the elements of $\mathscr{U}$.

(d) $G\left(X, X^{\prime}\right)$ : a nonvoid collection of homeomorphisms of $X$ onto itself, each carrying $X^{\prime}$ onto $X^{\prime} . G_{\mathscr{K}}$ will denote the collection of all homeomorphisms of $X$ onto $X$ each carrying $X^{\prime}$ onto $X^{\prime}$ and each supported on some element of $\mathscr{K}$. $G_{\mathscr{K}}^{*}$ will denote the group generated by $G_{\mathscr{X}}$.

If $X, X^{\prime}, \mathscr{K}$, and $G\left(X, X^{\prime}\right)$ are such as to satisfy the following axioms, then $\left(X, X^{\prime}, \mathscr{K}, G\left(X, X^{\prime}\right)\right)$ will be called an $A$-quadruple( $\left.{ }^{2}\right)$.

(1) If $K \in \mathscr{K}$, then $\mathrm{Cl}(\sim K) \in \mathscr{K}$.

(2) If $K \in \mathscr{K}$, and if $g \in G\left(X, X^{\prime}\right)$, then $g(K) \in \mathscr{K}$.

(3) If $g_{1}, g_{2} \in G\left(X, X^{\prime}\right)$, and if $g \in G_{\mathscr{X}}$, then $g g_{1}, g_{1} g \in G\left(X, X^{\prime}\right)$ and $g_{2} g_{1} \in G_{\mathscr{K}}^{*}$.

(4) If $K_{1}, K_{2}, K_{3} \in \mathscr{K}$, with $K_{1} \subset K_{3}^{0} \supset K_{2}$, then there exists an element $g \in G_{\mathscr{K}}$, supported on $K_{3}$, with $\left(g K_{1}\right)=K_{2}\left({ }^{3}\right)$.

(5) If $g \in G_{\mathscr{K}}^{*}$, and if $K_{1}, K_{2} \in \mathscr{K}$ with $K_{1} \subseteq K_{2}^{0}$ and $g\left(K_{1}\right)=K_{1}$, then there exists an element $g_{1} \in G_{\mathscr{K}}$, supported on $K_{2}$, such that $g_{1}\left|K_{1}=g\right| K_{1}$.

Axioms (1) and (2) will be frequently used and usually without being cited. Note that in the case where $X=X^{\prime}$ Axioms (1) and (4) assert a sort of invertibility for $X$.

Axiom (3) implies immediately that if $g \in G_{\mathscr{K}}^{*}$, and if $g_{1} \in G\left(X, X^{\prime}\right)$, then $g g_{1}, g_{1} g \in G\left(X, X^{\prime}\right)$. In view of this (3) also implies that if $g \in G\left(X, X^{\prime}\right)$, then $g^{-1} \in G\left(X, X^{\prime}\right)$ : for $g^{2} \in G_{\mathscr{X}}^{*}$; hence $g^{-2} \in G_{\mathscr{X}}^{*}$, and therefore $g^{-1}=g g^{-2} \in G(X, X)^{\prime}$.

It now follows that if $g \in G_{\mathscr{K}}^{*}$ and $K \in \mathscr{K}$, then $g(K) \in \mathscr{K}$. For since $G\left(X, X^{\prime}\right) \neq \varnothing$, there exists $g_{1} \in G\left(X, X^{\prime}\right)$. Then $g g_{1}^{-1} \in G\left(X, X^{\prime}\right)$, and $g_{1}(K) \in \mathscr{K}$ by Axiom (2). Hence, also by Axiom (2), $g(K)=g g_{1}^{-1}\left(g_{1}(K)\right) \in \mathscr{K}$.

Also Axiom (3) implies that if $g \in G\left(X, X^{\prime}\right)$ and $\delta \in G_{\mathscr{K}}^{*}$, then $\delta g \delta^{-1} \in G\left(X, X^{\prime}\right)$,i.e., the conjugate of any element of $G\left(X, X^{\prime}\right)$ by any element of $G_{\mathscr{X}}^{*}$ is an element of $G\left(X, X^{\prime}\right)$.

It follows also that the set $G\left(X, X^{\prime}\right)$ and $G_{\mathscr{X}}{ }^{*}$ are either equal or disjoint; or, in other words, $G\left(X, X^{\prime}\right)=G_{\mathscr{K}}^{*}$ if and only if $e \in G\left(X, X^{\prime}\right)$.

Note that since each of $G\left(X, X^{\prime}\right)$ and $G_{\mathscr{K}}^{*}$ consists solely of homeomorphisms which carry $X^{\prime}$ onto $X^{\prime}$, any homeomorphism which we may subsequently construct must satisfy this condition if it is to be in either $G\left(X, X^{\prime}\right)$ or $G_{\mathscr{X}}^{*}$.

Henceforth we shall assume that $\left(X, X^{\prime}, \mathscr{K}, G\left(X, X^{\prime}\right)\right)$ is an $A$-quadruple.

By way of example, while reading the arguments to follow, one might think of $X$ as the $n$-cell, $n>1$, and $X^{\prime}$ as its boundary.

Definition 1. Suppose $p \in X^{\prime}$ and $\mathscr{C}=\left\{C_{i}\right\}_{i=t_{0}}^{\infty} \subseteq \mathscr{K}$. Then $\mathscr{C}$ is called a null sequence with respect to $p$ or, more briefly, $\mathscr{C}$ is said to be null for $p$, if:

(a) $C_{i+1} \subset C_{i}^{0}, i \geqq t_{0}$, and

(2) This definition is due to R. D. Anderson [1] except that we omit his axiom two. Without using the concept of topological dilation he obtained previously Corollaries 3 and 4 to Theorem 2 .

(3) Do not forget that " $C$ " denotes proper containment, 
(b) $\left\{C_{l}^{0}\right\}_{i=t_{0}}^{\infty}$ is a neighborhood basis for $p$.

Of course if $\left\{C_{i}\right\}_{i=t_{0}}^{\infty}$ is null for $p$, then $p=\bigcap\left\{C_{i}\right\}_{i=t_{0}}^{\infty}$. Null sequences indexed from $t_{0}$ to $-\infty$ may be defined in a comparable fashion.

Definition 2. Suppose $p$ and $q$ are distinct points of $X^{\prime}$, and suppose $\mathscr{C}=\left\{C_{i}\right\}_{i=-\infty}^{\infty} \subseteq \mathscr{K}$. Then $\mathscr{C}$ is called a dilation structure with respect to the ordered pair $(p, q)$ provided:

(a) $\left\{C_{i}\right\}_{i=0}^{-\infty}$ is a null sequence for $p$, and

(b) $\left\{\mathrm{Cl}\left(\sim C_{i}\right)\right\}_{i=0}^{\infty}$ is a null sequence with respect to $q$.

Definition 3. Suppose $g \in G_{\mathscr{K}}^{*}$ and suppose $\mathscr{C}=\left\{C_{i}\right\}_{i=-\infty}^{\infty}$ is a dilation structure with respect to $(p, q)$. If $g\left(C_{i}\right)=C_{i+1},-\infty<i<\infty$, then $g$ is called a topological dilation with carrier $\mathscr{C}$; and $\mathscr{C}$ is said to carry $g$.

Note that if $\left\{C_{i}\right\}_{i=0}^{\infty}$ is null for $p$, and if $g \in G_{\mathscr{K}}^{*} \cup G\left(X, X^{\prime}\right)$, then $\left\{g\left(C_{i}\right)\right\}_{i=0}^{\infty}$ is null for $g(p)$. Hence if $g \in G_{\mathscr{X}}^{*}$ is a topological dilation with carrier $\mathscr{C}=\left\{C_{i}\right\}_{i=-\infty}^{\infty}$ with respect to $(p, q)$, and if $\delta \in G_{\mathscr{K}}^{*}$, then $\delta g \delta^{-1}$ is a topological dilation carried by $\mathscr{C}^{\prime}=\left\{\delta\left(C_{i}\right)\right\}_{i=-\infty}^{\infty}$ with respect to $(\delta(p), \delta(q))$.

2. Topological dilations. We begin this section by proving some lemmas which will be useful throughout the paper. Next we demonstrate the existence of topological dilations and, in fact, may conclude that $G_{\mathscr{X}}^{*}$ is richly supplied with them. We conclude $\$ 2$ with Theorem 1 , which asserts any two topological dilations are conjugate in $G_{\mathscr{X}}^{*}$.

Proposition A. Suppose $p$ and $q$ are distinct points of $X^{\prime}$. Then there exists a dliation structure with respect to $(p, q)$.

Proof. Since $X$ is first countable, regular, and Hausdorff, $X^{\prime}$ is perfect, and $\mathscr{U}$ is a basis, it easily follows that we may find sequences of elements of $\mathscr{K}$, $\left\{C_{i}\right\}_{i=0}^{-\infty}$ and $\left\{D_{i}\right\}_{i=1}^{\infty}$, null for $p$ and $q$ respectively, with $C_{0} \cap D_{1}=\varnothing$ and $C_{0} \cup D_{1} \neq X$. Setting $C_{i}=\mathrm{Cl}\left(\sim D_{i}\right)$ for $i \geqq 1$, we see that $\mathscr{C}=\left\{C_{i}\right\}_{i=-\infty}^{\infty}$ is the desired dilation structure.

Proposition B. Suppose $K_{1}, K_{2} \in \mathscr{K}$ and suppose $K_{1} \subset K_{2}^{0}$. Then there exists $K \in \mathscr{K}$ with $K_{1} \subset K^{0}$ and $K \subset K_{2}^{0}$.

Proof. Select $x \in K_{1}^{0} \cap X^{\prime}$ and $E_{1} \in \mathscr{K}$ with $x \in E_{1}^{0}$ and $E_{1} \subset K_{1}^{0}$. This can be done since $X$ is regular. Now select $E_{2} \in \mathscr{K}$, with $x \in E_{2}^{0}, E_{2} \subset E_{1}^{0}$. Using Axiom (4), we obtain $g \in G_{\mathscr{K}}$, supported on $K_{2}$, with $g\left(K_{1}\right)=E_{2}$. Set $K=g^{-1}\left(E_{1}\right)$.

Lemma 1. Suppose $K \in \mathscr{K}$ and $x_{0}, y_{0} \in\left(K^{0} \cap X^{\prime}\right)$; suppose also that $\mathscr{C}=\left\{C_{i}\right\}_{i=1}^{\infty}, \mathscr{D}=\left\{D_{i}\right\}_{i=1}^{\infty} \subseteq \mathscr{K}$ are null for $x_{0}, y_{0}$ respectively, with $C_{1} \subset K^{0} \supset D_{1}$. Then there is a homeomorphism $h \in G_{\mathscr{K}}$, supported on $K$, such that $h\left(C_{i}\right)=D_{i}$ for $i \geqq 1$.

Proof. By Axiom (4) there exists $h_{1} \in G_{\mathscr{X}}$, supported on $K$, such that $h_{1}\left(C_{1}\right)=D_{1}$. For $i \geqq 2$ we define inductively, by making repeated use of Axiom (4), $h_{i} \in G_{\mathscr{X}}$, supported on $D_{i-1}$, such that $h_{i} h_{i-1} \cdots h_{1}\left(C_{i}\right)=D_{i}$. 
Set $h=\prod_{i=1}^{\infty} h_{i}$. If $x \neq x_{0}$, then for some $j \geqq 1, x \notin C_{j}$. Then $\prod_{i=1}^{j} h_{i}(x) \notin D_{j}=\prod_{i=1}^{j} h_{i}\left(C_{j}\right)$. Since for $i>j, h_{i}$ is supported on $D_{j}$, it follows that $h(x)=\prod_{i=1}^{j} h_{i}(x)$. From this, since $h_{i} \in G_{\mathscr{X}}$ and $\mathscr{C}$ and $\mathscr{D}$ are null for $x_{0}$ and $y_{0}$ respectively, it follows that $h$ is a homeomorphism supported on $K, h\left(x_{0}\right)=y_{0}$, and $h$ takes $X^{\prime}$ onto $X^{\prime}$. Hence $h \in G_{\mathscr{K}}$. As a corollary we obtain Lemma 2, which resembles Anderson's telescoping Lemma [1].

Lemma 2. Suppose $K_{0}, K_{1} \in \mathscr{K}$ with $K_{1} \subseteq K_{0}^{0}$ and $g \in G\left(X, X^{\prime}\right)$ with $g\left(K_{0}\right) \subset K_{1}^{0}$. Suppose also that $x_{0} \in K_{1}^{0} \cap X^{\prime},\left\{D_{i}\right\}_{i=1}^{\infty}$ is null for $x_{0}$ with $D_{1} \subset K_{1}^{0}$. Then there exists $h \in G_{\mathscr{X}}$, supported on $K_{1}$, such that $(h g)^{i}\left(K_{0}\right)=D_{i}$, for $i \geqq 1$.

Proof. Set $C_{1}=g\left(K_{0}\right)$ and $C_{i}=g\left(D_{i-1}\right)$ for $i \geqq 2$. Then $\left\{C_{i}\right\}_{i=1}^{\infty}$ is null for $g\left(x_{0}\right)$. Hence Lemma 1 asserts the existence of $h \in G_{\mathscr{K}}$, supported on $K_{1}$, such that $h\left(C_{i}\right)=D_{i}$, for $i \geqq 1$. Hence $(h g)^{i}\left(K_{0}\right)=D_{i}$, for $i \geqq 1$.

Lemma 3. Any dilation structure carries a topological dilation.

Proof. Suppose $\mathscr{B}=\left\{B_{i}\right\}_{i=-\infty}^{\infty}$ is a dilation structure with respect to $(p, q)$. By Proposition B there is an element $K_{1} \in \mathscr{K}$ such that $B_{0} \subset K_{1}^{0}, K_{1} \subset B_{1}^{0}$. We now apply Lemma 1 with $K_{1}$ as $K, B_{-i}$ as $C_{i}$, and $B_{-i+1}$ as $D_{i}$ for $i \geqq 1$, and $x_{0}=p=y_{0}$ to obtain $h_{1} \in G_{\mathscr{X}}$, supported on $K_{1}$, with $h_{1}\left(B_{-i}\right)=B_{-i+1}$, for $i \geqq 1$.

Since $B_{0} \subset h_{1}\left(B_{0}^{0}\right)$, we may find $K_{2} \in \mathscr{K}$ with $B_{0} \subset K_{2}^{0}, K_{2} \subset h_{1}\left(B_{0}^{0}\right)$. Then $\mathrm{Cl}\left(\sim h_{1}\left(B_{0}\right)\right) \subset \sim K_{2}$, and $\mathrm{Cl}\left(\sim B_{i}\right) \subset \sim K_{2}, i \geqq 1$. So we again apply Lemma 1 to obtain $h_{2} \in G_{\mathscr{X}}$, supported on $\mathrm{Cl}\left(\sim K_{2}\right)$ with $h_{2}\left(\mathrm{Cl}\left(\sim h_{1}\left(B_{0}\right)\right)\right)=\mathrm{Cl}\left(\sim B_{1}\right)$, and $h_{2}\left(\mathrm{Cl}\left(\sim B_{i}\right)\right)=\mathrm{Cl}\left(\sim B_{i+1}\right), i \geqq 1$.

$h=h_{2} h_{1} \in G_{\mathscr{K}}^{*}$ is the desired topological dilation.

COROLlaRy. There exists an element $g \in G_{\mathscr{X}}^{*}$ such that $g$ is a topological dilation.

Proof. Since $X^{\prime}$ is perfect, $X^{\prime}$ contains two distinct points. The corollary now follows from Proposition A and Lemma 3.

We remarked earlier that if $g \in G_{\mathscr{K}}^{*}$ is an arbitrary topological dilation, then any conjugate of $g$ by an element of $G_{\mathscr{K}}^{*}$ is also a topological dilation. Theorem 1 asserts that the converse is true: that any topological dilation is a conjugate of $g$.

THEOREM 1. Suppose $g_{1}$ and $g_{2}$ are topological dilations. Then there is an element $r$ of $G_{\mathscr{X}}^{*}$ such that $g_{1}=r g_{2} r^{-1}$.

Proof. Let $\mathscr{C}=\left\{C_{i}\right\}_{i=-\infty}^{\infty}$ be a dilation structure with respect to $(p, q)$; and let $\mathscr{D}^{\prime}=\left\{D_{i}^{\prime}\right\}_{i=-\infty}^{\infty}$ be a dilation structure with respect to $\left(p^{\prime}, q^{\prime}\right)$; with $\mathscr{C}$ and $\mathscr{D}$ carrying $g_{2}$ and $g_{1}$ respectively.

We may select $a \in \sim C_{0} \cap X^{\prime} a \neq p^{\prime}$. There is an integer $i_{0} \leqq 0$ such that $a \notin D_{i_{0}}^{\prime}$. Hence we may find $K_{1} \in \mathscr{K}$ with $a \in K_{1}^{0}, K_{1} \cap\left(C_{0} \cup D_{i_{0}}^{\prime}\right)=\varnothing$, and $\sim K_{1} \neq\left(C_{0} \cup D_{i_{0}}\right)$. Set $D_{i}=D_{i+i_{0}},-\infty<i<\infty ;$ then $\mathscr{D}=\left\{D_{i}\right\}_{i=-\infty}^{\infty}$ carries $g_{1}$, and $D_{0}=D_{i_{0}}$. 
Since $C_{0} \cup D_{0} \subset \sim K_{1}$, we may invoke Lemma 1 to obtain $\delta_{1} \in G_{\mathscr{X}}$, supported on $\mathrm{Cl}\left(\sim K_{1}\right)$, with $\delta_{1}\left(C_{i}\right)=D_{i}, i \leqq 0$.

$\left\{\delta_{1}\left(\mathrm{Cl}\left(\sim C_{i}\right)\right)\right\}_{i=1}^{\infty}$ is null for $\delta_{1}(q)$, and $\delta_{1}\left(\mathrm{Cl}\left(\sim C_{0}\right)\right)=\mathrm{Cl}\left(\sim D_{0}\right)$. Hence we may again apply Lemma 1 , this time to the sequences $\left\{\delta_{1}\left(\mathrm{Cl}\left(\sim C_{i}\right)\right)\right\}_{i=1}^{\infty}$ and $\left\{\mathrm{Cl}\left(\sim D_{i}\right)\right\}_{i=1}^{\infty}$, to obtain $\delta_{2} \in G_{\mathscr{K}}$, supported on $\mathrm{Cl}\left(\sim D_{0}\right)$, with $\delta_{2} \delta_{1}\left(\mathrm{Cl}\left(\sim C_{i}\right)\right)$ $=\mathrm{Cl}\left(\sim D_{i}\right), i \geqq 1$.

Set $\delta=\delta_{2} \delta_{1} \in G_{\mathscr{X}}^{*} \cdot \delta\left(C_{i}\right)=D_{i},-\infty<i<\infty$, and $\delta(p)=p^{\prime}, \delta(q)=q^{\prime}$. Hence $\mathscr{D}$ carries the topological dilation $g=\delta g_{2} \delta^{-1}$.

We may think of the construction of $\delta$ as the first step in the conjugation procedure to change $g_{2}$ into $g_{1}$, since it yields the topological dilation $g$ which is carried by the same structure that carries $g_{1}$. Of course pointwise $g$ need not equal $g_{1}$. To achieve pointwise equality a further conjugation in necessary, which in the event that $D_{0}$ is open and closed is quite simple. We proceed to that case:

Set $B_{i}=$ (boundary of $\left.D_{i}\right)=D_{i}-D_{i}^{0}$. Since $g^{i}\left(B_{0}\right)=B_{i},-\infty<i<\infty, B_{i}=\varnothing$. if and only if $B_{0}=\varnothing$. If $B_{0}=\varnothing$, define $\alpha$ as follows:

$$
\alpha\left(p^{\prime}\right)=p^{\prime}, \quad \alpha\left(q^{\prime}\right)=q^{\prime} ;
$$

if $x \in D_{i+1}-D_{i}, \alpha(x)=g_{1}^{i} g^{-i}(x),-\infty<i<\infty$.

Then $\alpha$ is a homeomorphism of $X$ onto itself taking $X^{\prime}$ onto $X^{\prime}$. Since $\alpha$ takes $D_{0}$ onto itself, and since $B_{0}=\varnothing$, if we define $\alpha_{1}=\alpha$ on $D_{0}$ and $\alpha_{1}=e$ on $\sim D_{0}$; and $\alpha_{2}=\alpha$ on $\sim D_{0}$ and $\alpha_{2}=e$ on $D_{0}$, then $\alpha_{1}, \alpha_{2} \in G_{\mathscr{X}}$ and $\alpha=\alpha_{2} \alpha_{1} \in G_{\mathscr{X}}^{*}$. Since $g_{1}=\alpha g \alpha^{-1}$, if we set $r=\alpha \delta$, then $g_{1}=r g_{2} r^{-1}$.

It is in the remaining case that Axiom (5) finds its only employment. Thus we might observe now, if the reader hasn't already done so, that in case $\mathscr{K}$ consists of sets which are open and closed in $X$, Axiom (5) may be omitted.

Now suppose $B_{0} \neq \varnothing$.

By Proposition $\mathrm{B}$ there exists $K_{1} \in \mathscr{K}$ with $D_{0} \subset K_{1}^{0}, K_{1} \subset D_{1}^{0}$. Set $\alpha_{1}=g_{1} g^{-1} \in G_{\mathscr{X}}^{*} ; \alpha_{1}$ takes $D_{i}$ onto $D_{i},-\infty<i<\infty$. By Axiom (5) there exists $\bar{\alpha}_{1} \in G_{\mathscr{X}}$, supported on $\mathrm{Cl}\left(\sim K_{1}\right)$ such that $\bar{\alpha}_{1} \mid \mathrm{Cl}\left(\sim D_{1}\right)=\alpha_{1}$. In particular, $\bar{\alpha}_{1}$ takes $D_{i}$ onto $D_{i}$ and $\bar{\alpha}_{1} \mid B_{1}=\alpha_{1}$.

Proceeding inductively, for $i \geqq 2$ we may select $K_{i} \in \mathscr{K}$ with $D_{i-1} \subset K_{i}^{0}, K_{i} \subset D_{i}^{0}$. Set

$$
\alpha_{i}=g_{1}^{i} g^{-i}\left(\bar{\alpha}_{i-1} \cdot \bar{\alpha}_{i-2} \cdots \bar{\alpha}_{1}\right)^{-1} \text {. }
$$

$\alpha_{i} \in G_{\mathscr{X}}^{*}$ and $\alpha_{i}$ takes $D_{j}$ onto $D_{j},-\infty<j<\infty$. By Axiom (5) there is $\bar{\alpha}_{i} \in G_{\mathscr{K}}$, supported on $\mathrm{Cl}\left(\sim K_{i}\right)$, such that $\bar{\alpha}_{i} \mid \mathrm{Cl}\left(\sim D_{i}\right)=\alpha_{i}$. In particular, $\bar{\alpha}_{i}$ takes $D_{j}$ onto $D_{j}$ and $\bar{\alpha}_{i} \mid B_{i}=\alpha_{i}$.

Set $\alpha^{\prime}=\prod_{i=1}^{\infty} \bar{\alpha}_{i} . \alpha^{\prime}$ is a homeomorphism, supported on $\mathrm{Cl}\left(\sim K_{1}\right)$, and taking $X^{\prime}$ onto $X^{\prime}$ and $X$ onto $X$; hence $\alpha^{\prime} \in G_{\mathscr{X}}$.

Observe that $\alpha^{\prime} \mid B_{i}=g_{1}^{i} g^{-i}, i \geqq 0$; and $\alpha^{\prime}$ takes $D_{i}$ onto $D_{i},-\infty<i<\infty$.

Now take $K_{-1} \in \mathscr{K}$ with $D_{-1} \subset K_{-1}^{0}, K_{-1} \subset D_{0}^{0}$. Set $\alpha_{-1}=g_{1}^{-1} g . \alpha_{-1} \in G_{\mathscr{K}}^{*}$, 
and $\alpha_{-1}$ takes $D_{i}$ onto $D_{i},-\infty<i<\infty$. As before, from Axiom (5) we obtain $\bar{\alpha}_{-1} \in G_{\mathscr{X}}$, supported on $K_{-1}$, with $\bar{\alpha}_{-1} \mid D_{-1}=\alpha_{-1}$. In particular, $\bar{\alpha}_{-1}$ takes $D_{i}$ onto $D_{i}$, and $\bar{\alpha}_{-1} \mid B_{-1}=\alpha_{-1}$.

Again proceeding inductively for $i \geqq 2$, select $K_{-i} \in \mathscr{K}$ with $D_{-i} \subset K_{-i}^{0}$, $K_{-i} \subset D_{-i+1}^{0}$. Set

$$
\alpha_{-i}=g_{1}^{-i} g^{i}\left(\bar{\alpha}_{-i+1} \cdot \bar{\alpha}_{-i+2} \cdots \bar{\alpha}_{-1}\right)^{-1} \text {. }
$$

Then $\alpha_{-i}$ takes $D_{j}$ onto $D_{j},-\infty<j<\infty$, and $\alpha_{-i} \in G_{\mathscr{X}}^{*}$. We use Axiom (5) to obtain $\bar{\alpha}_{-i} \in G_{\mathscr{K}}$, supported on $K_{-i}$, with $\bar{\alpha}_{-i} \mid D_{-i}=\alpha_{-i}$. And $\bar{\alpha}_{-i}$ takes $D_{j}$ onto $D_{j}$, and $\bar{\alpha}_{-i} \mid B_{-i}=\alpha_{-i}$.

Set $\bar{\alpha}=\prod_{i=1}^{\infty} \bar{\alpha}_{-i} . \bar{\alpha} \in G_{\mathscr{K}}$, is supported on $K_{-1}$, takes $D_{i}$ onto $D_{i},-\infty<i<\infty$, and $\bar{\alpha} \mid B_{-i}=g_{1}^{-i} g^{i}, i \geqq 0$.

Now set $\alpha=\bar{\alpha} \cdot \alpha^{\prime} \in G_{\mathscr{X}}^{*}$. Since $K_{-1} \cap \mathrm{Cl}\left(\sim K_{1}\right)=\varnothing, \alpha$ takes $D_{i}$ onto $D_{i}$ and $\alpha \mid B_{i}=g_{1}^{i} g^{-i},-\infty<i<\infty$.

Setting $\bar{g}=\alpha g \alpha^{-1}$, we observe that $\bar{g} \mid B_{i}=g_{1},-\infty<i<\infty$.

Finally, we define $\beta$ as follows:

$$
\beta\left(p^{\prime}\right)=p^{\prime}, \quad \beta\left(q^{\prime}\right)=q^{\prime} ;
$$

if $x \in D_{i+1}-D_{i}, \beta(x)=g_{1}^{i} \bar{g}^{-i}(x),-\infty<i<\infty$.

$\beta$ is a homeomorphism of $X$ onto itself, carrying $X^{\prime}$ onto $X^{\prime}$. Also $\beta \mid B_{i}=e$, $-\infty<i<\infty$. And since, in addition, $\beta$ takes $D_{0}$ onto $D_{0}$, if we define $\beta_{1}=\beta$ on $D_{0}$ and $\beta_{1}=e$ on $\sim D_{0}$; and $\beta_{2}=\beta$ on $\sim D_{0}$ and $\beta_{2}=e$ on $D_{0}$, then $\beta_{1}, \beta_{2} \in G_{\mathscr{K}}$; hence $\beta=\beta_{2} \beta_{1} \in G_{\mathscr{K}}^{*}$. And since $g_{1}=\beta \bar{g} \beta^{-1}$, if we set $r=\beta \alpha \delta \in G_{\mathscr{K}}^{*}$, we have $g_{1}=r g_{2} r^{-1}$.

3. The principal theorem; its corollaries. In this section we prove the principal theorem of the paper and deduce some corollaries.

THEOREM 2. Suppose $f$ and $g$ are elements of $G\left(X, X^{\prime}\right)$ neither of which is the identity on $X^{\prime}$. Then there exists elements $h_{1}$ and $h_{2}$ of $G_{\mathscr{X}}^{*}$ such that $\left(h_{2} g h_{2}^{-1}\right)\left(h_{1} f h_{1}^{-1}\right)$ is a topological dilation. Furthermore $h_{1}$ and $h_{2}$ may be chosen as topological dilations.

Proof. We distinguish two cases: Case 1. At least one of $f$ and $g$, say $f$, is not of period two on $X^{\prime}$. Case 2. Both $f$ and $g$ are of period two on $X^{\prime}$.

Case 1. Since $f$ is not of period two on $X^{\prime}$, neither $f$ nor $g$ is the identity on $X^{\prime}$, and $X^{\prime}$ is perfect, we may find elements $C_{1}, D_{1}, A_{0}$, and $B_{0}$ of $\mathscr{K}$ such that the sets $\left\{C_{1}, D_{1}, A_{0}, B_{0}, f\left(A_{0}\right), f^{-1}\left(A_{0}\right), g\left(B_{0}\right)\right\}$ are mutually disjoint and such that the sets $\left\{C_{1}, D_{1}, A_{0}, B_{0}, f\left(A_{0}\right), f^{-1}\left(A_{0}\right), g^{-1}\left(B_{0}\right)\right\}$ are mutually disjoint.

Select $c \in C_{1}^{0} \cap X^{\prime}, d \in D_{1}^{0} \cap X^{\prime}, a \in A_{0}^{0} \cap X^{\prime}$, and $b \in B_{0}^{0} \cap X^{\prime}$. We now take sequences of elements of $\mathscr{K}-\left\{C_{i}\right\}_{i=1}^{\infty},\left\{D_{i}\right\}_{i=1}^{\infty},\left\{A_{i}\right\}_{i=0}^{-\infty}$, and $\left\{B_{i}\right\}_{i=0}^{-\infty}$-null for $c$, $d, a$, and $b$ respectively.

Set $A_{i}=\mathrm{Cl}\left(\sim C_{i}\right), B_{i}=\mathrm{Cl}\left(\sim D_{i}\right), i \geqq 1$. Then $\mathscr{A}=\left\{A_{i}\right\}_{i=-\infty}^{\infty}$ and $\mathscr{B}=\left\{B_{i}\right\}_{i=-\infty}^{\infty}$ 
are dilation structures with respect to $(a, c)$ and $(b, d)$ respectively. By Lemma 3 $\mathscr{A}$ and $\mathscr{B}$ respectively carry topological dilations $\delta$ and $r$.

Set $\beta=\left(r g r^{-1}\right)\left(\delta f \delta^{-1}\right) . \beta \in G_{\mathscr{X}}^{*}$ by Axiom (3).

Now a remark about what is to follow: It is true that $\beta\left(A_{1}\right) \subset A_{1}^{0}$ and $\beta^{-1}\left(C_{1}\right) \subset C_{1}^{0}\left(A_{1}=\mathrm{Cl}\left(\sim C_{1}\right)\right)$. Hence $\beta^{i}\left(A_{1}\right) \subset \beta^{i-1}\left(A_{1}^{0}\right)$ for $i \geqq 1$, and $\beta^{-i}\left(C_{1}\right)$ $\subset \beta^{-i+1}\left(C_{1}^{0}\right), i \geqq 1$. Therefore if $\left\{\beta^{i}\left(A_{1}\right)\right\}_{i=0}^{\infty}$ were null for a point of $X^{\prime}$ and if $\left\{\beta^{-i}\left(C_{1}\right)\right\}_{i=0}^{\infty}$ were null for a point of $X^{\prime}$, then it is apparent that $\beta$ would then be a topological dilation. Therefore, in order to achieve this we are going to multiply $r$ and $\delta$ by appropriate elements of $G_{\mathscr{K}}^{*}$.

Consider $\beta\left(A_{1}\right)$ :

$$
\begin{aligned}
\delta^{-1}\left(A_{1}\right) & =A_{0}, \\
f\left(A_{0}\right) & \subset A_{1}^{0}-A_{0}, \\
\delta\left(A_{1}^{0}-A_{0}\right) & =A_{2}^{0}-A_{1}=C_{1}^{0}-C_{2} .
\end{aligned}
$$

Hence

$$
\begin{aligned}
\delta f \delta^{-1}\left(A_{1}\right) & \subset C_{1}^{0}-C_{2}, \\
r^{-1}\left(C_{1}\right) & \subset B_{0}^{0}-B_{-1}, \\
g\left(B_{0}\right) & \subset B_{1}^{0}-B_{0} \\
r\left(B_{1}^{0}-B_{0}\right) & =B_{2}^{0}-B_{1}=D_{1}^{0}-D_{2} .
\end{aligned}
$$

Hence $\beta\left(A_{1}\right) \subset D_{1}^{0}-D_{2}$. Set $K_{1}=r g\left(B_{0}\right) . \beta\left(A_{1}\right) \subset K_{1}^{0}, K_{1} \subset D_{1}^{0}-D_{2}$. Select $x_{1} \in K_{1}^{0} \cap X^{\prime}$ and a sequence of elements of $\mathscr{K},\left\{T_{i}\right\}_{i=1}^{\infty}$, null for $x_{1}$, with $T_{1} \subset K_{1}^{0}$. We now apply Lemma 2 , with $A_{1}$ as $K_{0}$ and with $\beta$ as $g$, to obtain $\alpha_{1} \in G_{\mathscr{K}}$, supported on $K_{1}$, with $\left(\alpha_{1} \beta\right)^{i}\left(A_{1}\right)=T_{i}, i \geqq 1$.

Now set $\bar{\beta}=\left(\delta f^{-1} \delta^{-1}\right)\left(\alpha_{1} r g^{-1} r^{-1} \alpha_{1}^{-1}\right)$ and consider $\bar{\beta}\left(C_{1}\right)$.

Since $K_{1} \cap C_{1}=\varnothing, r g^{-1} r^{-1} \alpha_{1}^{-1}\left(C_{1}\right)=r g^{-1} r^{-1}\left(C_{1}\right)$,

$$
\begin{aligned}
r^{-1}\left(C_{1}\right) & \subset B_{0}^{0}, \\
g^{-1}\left(B_{0}\right) & \subset B_{1}^{0}-B_{0}, \\
r\left(B_{1}^{0}-B_{0}\right) & =B_{2}^{0}-B_{1}=D_{1}^{0}-D_{2} .
\end{aligned}
$$

So $\mathrm{rg}^{-1} r^{-1}\left(C_{1}\right) \subset D_{1}^{0}-D_{2}$. Since $K_{1} \subset D_{1}^{0}-D_{2}$,

$$
\begin{aligned}
\alpha_{1} r g^{-1} r^{-1}\left(C_{1}\right) & \subset D_{1}^{0}-D_{2} \subset D_{1}^{0}, \\
\delta^{-1}\left(D_{1}\right) & \subset A_{0}^{0} ; \text { and } f^{-1}\left(A_{0}\right) \subset A_{1}^{0}-A_{0}, \\
\delta\left(A_{1}^{0}-A_{0}\right) & =A_{2}^{0}-A_{1}=C_{1}^{0}-C_{2} .
\end{aligned}
$$

Hence $\bar{\beta}\left(C_{1}\right) \subset C_{1}^{0}-C_{2}$.

Set $K_{2}=\delta f^{-1}\left(A_{0}\right)$. Then $\bar{\beta}\left(C_{1}\right) \subset K_{2}^{0}, K_{2} \subset C_{1}^{0}-C_{2}$. Note that $K_{2} \cap \delta f\left(A_{0}\right)=\varnothing$. 
Select $x_{2} \in K_{2}^{0} \cap X^{\prime}$ and a sequence of elements of $\mathscr{K},\left\{T_{i}\right\}_{i=-1}^{-\infty}$ null for $x_{2}$, with $T_{-1} \cap K_{2}^{0}$. We again apply Lemma 2 , this time with $C_{1}$ as $K_{0}$ and with $\bar{\beta}$ as $g$, to obtain $\alpha_{2} \in G_{\mathscr{K}}$, supported on $K_{2}$, with $\left(\alpha_{2} \bar{\beta}\right)_{i}\left(C_{1}\right)=T_{-i}, i \geqq 1$.

Set $d=\left(\alpha_{1} r g r^{-1} \alpha_{1}^{-1}\right)\left(\alpha_{2} \delta f \delta^{-1} \alpha_{2}^{-1}\right)$ and observe that if $d^{i}\left(A_{1}\right)=T_{i}$ and $d^{-i}\left(C_{1}\right)=T_{-i}$ for $i \geqq 1$, then $d$ is a topological dilation carried by the dilation structure $\left\{T_{i}^{\prime}\right\}_{i=-\infty}^{\infty}$ with respect to $\left(x_{2}, x_{1}\right)$, where $T_{i}^{\prime}=T_{i}, i \leqq-1, T_{0}^{\prime}=C_{1}$, $T_{i}^{\prime}=\mathrm{Cl}\left(\sim T_{i}\right), i \geqq 1$.

Consider $d\left(A_{1}\right)$ :

Since $K_{2} \cap A_{1}=\varnothing, \delta f \delta^{-1} \alpha_{2}^{-1}\left(A_{1}\right)=\delta f \delta^{-1}\left(A_{1}\right)=\delta f\left(A_{0}\right)$. Since $K_{2} \cap \delta f\left(A_{0}\right)$ $=\varnothing, \alpha_{2} \delta f\left(A_{0}\right)=\delta f\left(A_{0}\right) \subset C_{1}$. But $K_{1} \cap C_{1}=\varnothing$; so $\alpha_{1}^{-1} \delta f\left(A_{0}\right)=\delta f\left(A_{0}\right)$. Hence $d\left(A_{1}\right)=\alpha_{1} \operatorname{rgr}^{-1} \delta f \delta^{-1}\left(A_{1}\right)=\alpha_{1} \beta\left(A_{1}\right)=T_{1}$. Since for $i \geqq 1, T \subset A_{1}$, $d\left(T_{i}\right)=\alpha_{1} \beta\left(T_{i}\right)=T_{i+1}$. Therefore $d^{-i}\left(A_{1}\right)=T_{-i}, i \geqq 1$.

Now consider $d^{-1}\left(C_{1}\right)=\alpha_{2} \delta f^{-1} \delta^{-1} \alpha_{2}^{-1} \alpha_{1} r g^{-1} r^{-1} \alpha_{1}^{-1}\left(C_{1}\right)$ :

As seen earlier, $\alpha_{1} r g^{-1} r^{-1} \alpha_{1}^{-1}\left(C_{1}\right) \subset D_{1}^{0}$. Since $K_{2} \cap D_{1}=\varnothing$,

$$
\alpha_{2}^{-1} \alpha_{1} r g^{-1} r^{-1} \alpha_{1}^{-1}\left(C_{1}\right)=\alpha_{1} r g^{-1} r^{-1} \alpha_{1}^{-1}\left(C_{1}\right) \text {. }
$$

Hence $d^{-1}\left(C_{1}\right)=\alpha_{2} \delta f^{-1} \delta^{-1} \alpha_{1} r^{-1} r^{-1} \alpha_{1}^{-1}\left(C_{1}\right)=\alpha_{2} \bar{\beta}\left(C_{1}\right)=T_{-1}$. And since for $i \geqq 1, T_{-i} \subset C_{1}, d^{-1}\left(T_{-i}\right)=\alpha_{2} \bar{\beta}\left(T_{-i}\right)=T_{-i-1}$. Hence $d^{-i}\left(C_{1}\right)=T_{-i}$, and hence $d$ is a topological dilation.

Furthermore, since $\alpha_{1}$ is supported on $K_{1} \subset D_{1}^{0}-D_{2}$ and $\alpha_{2}$ is supported on $K_{2} \cap C_{1}^{0}-C_{2}, h_{1}=\alpha_{2} \delta$ and $h_{2}=\alpha_{1} r$ are topological dilations carried by $\mathscr{A}$ and $\mathscr{B}$ respectively; and $d=\left(h_{2} g h_{2}^{-1}\right)\left(h_{1} f h_{1}^{-1}\right)$.

In the above we had set $K_{2}=\delta f^{-1}\left(A_{0}\right)$ and had then remarked that $K_{2} \subset \delta f\left(A_{0}\right)=\varnothing$. It was this that enabled us to construct $\alpha_{1}$ independently of $\alpha_{2}$. Of course the construction of $\alpha_{2}$ was dependent on $\alpha_{1}$; however we defined $K_{1}$ $=r g\left(B_{0}\right)$ and had we been able to assert that $K_{1} \cap r g^{-1}\left(B_{0}\right)=\varnothing$ we could have constructed $\alpha_{1}$ and $\alpha_{2}$ independently of each other. But, since in the event that $g$ was of period two on $X^{\prime}, K_{1} \cap \mathrm{rg}^{-1}\left(B_{0}\right) \neq \varnothing$, no such assertion was possible. Had $f$ also been of period two on $X^{\prime}$ then neither $\alpha_{1}$ nor $\alpha_{2}$ could have been constructed independently of the other. For this reason Case 2 requires a slightly modified procedure.

Case 2. Since neither $f$ nor $g$ is the identity on $X^{\prime}$, we may select elements of $\mathscr{K}, C_{0}, D_{0}, A_{0}$, and ${ }^{*} B_{0}$, such that the sets $\left\{C_{0}, D_{0}, A_{0},{ }^{*} B_{0}, f\left(A_{0}\right), g\left({ }^{*} B_{0}\right)\right\}$ are mutually disjoint and the sets $\left\{C_{0}, D_{0}, A_{0},{ }^{*} B_{0}, f^{-1}\left(A_{0}\right), g^{-1}\left({ }^{*} B_{0}\right)\right\}$ are mutually disjoint.

Now select $c \in C_{0}^{0} \cap X^{\prime}, d \in D_{0}^{0} \cap X^{\prime}, a \in A_{0}^{0} \cap X^{\prime}$, and $b \in{ }^{*} B_{0}^{0} \cap X^{\prime}$. As before, we may select null sequences $\left\{C_{i}\right\}_{i=0}^{\infty},\left\{D_{i}\right\}_{i=0}^{\infty},\left\{A_{i}\right\}_{i=0}^{-\infty}$, and $\left\{B_{i}\right\}_{i=0}^{-\infty}$ for $c, d, a$, and $b$ respectively with $B_{0} \subset{ }^{*} B_{0}^{0}$.

Wenow set $A_{i}=\mathrm{Cl}\left({ }^{\sim} C_{i}\right), B_{i}=\mathrm{Cl}\left({ }^{\sim} D_{i}\right)$ for $i \geqq 1$ and observe that $\mathscr{A}=\left\{A_{i}\right\}_{i=-\infty}^{\infty}$ and $\mathscr{B}=\left\{B_{i}\right\}_{i=-\infty}^{\infty}$ are dilation structures with respect to $(a, c)$ and $(b, d)$ respectively and hence, by Lemma 3 , carry topological dilations $\delta_{1}$ and $r_{1}$ respectively. 
$r_{1}^{-1}\left(C_{0}\right) \subset B_{0}^{0}-B_{1}$ and, since $g r_{1}^{-1}(c)=g^{-1} r_{1}^{-1}(c) \in g\left(B_{0}^{0}\right)$, we may select $N \in \mathscr{K}$ with $r_{1}^{-1}(c) \in N \subset r_{1}^{-1}\left(C_{0}^{0}\right)$ and such that $g^{-1}(N) \subset g\left(B_{0}^{0}\right)$. Since $C_{1} \subset C_{0}^{0} \supset r_{1}(N)$, we may invoke Axiom (4) to obtain $\alpha_{1} \in G_{\mathscr{X}}$, supported on $C_{0}$, with $\alpha_{1} r_{1}(N)=C_{1}$.

Set $r=\alpha_{1} r_{1}$ and observe that $r$ is a topological dilation carried by $\mathscr{B}$ and $r(N)=C_{1}$.

Employing an identical procedure, we can obtain $K_{1} \in \mathscr{K}$, and $\alpha_{2} \in G_{\mathscr{K}}$ such that $\alpha_{2}$ is supported on $D_{0}, K_{1} \subset A_{0}^{0}-A_{-1}, f^{-1}\left(K_{1}\right) \subset f\left(A_{0}^{0}\right)$, and $\alpha_{2} \delta_{1}\left(K_{1}\right)=D_{1}$.

Set $\delta=\alpha_{2} \delta_{1}$ and note that $\delta$ is a topological dilation carried by $\mathscr{A}$ and $\delta\left(K_{1}\right)=D_{1}$.

Now $r g(b) \in r g\left({ }^{*} B_{0}\right) \subset D_{1}^{0}-D_{2}$. Hence $\delta^{-1} r g(b) \in K_{1}^{0}$. Set $x_{0}=\delta^{-1} r g(b)$. Since $g^{-1} r^{-1} \delta f^{-1}\left(x_{0}\right)=g r^{-1} \delta f\left(x_{0}\right)$, we may select $\left\{K_{i}\right\}_{i=2}^{\infty}$, null for $x_{0}$, with $K_{2} \subset K_{1}^{0}$, such that $g^{-1} r^{-1} \delta f^{-1}\left(K_{i}\right) \subset g r^{-1} \delta f\left(K_{i-1}^{0}\right)$ for $i \geqq 2$ and with $\delta\left(K_{2}\right) \subset r g\left({ }^{*} B_{0}^{0}\right)$.

Set $E_{i}=r^{-1} \delta f^{-1}\left(K_{i}\right), i \geqq 2$. Then $r\left(E_{i}\right)=\delta f^{-1}\left(K_{i}\right)$ and $g^{-1}\left(E_{i}\right) \subset g r^{-1} \delta f\left(K_{i-1}^{0}\right)$, $i \geqq 2$.

Now define $\pi \in G_{\mathscr{X}}^{*}$ as: $\pi=r g r^{-1} \delta f \delta^{-1}$.

Consider $\pi\left(A_{1}\right): \delta^{-1}\left(A_{1}\right)=A_{0}$, so $\delta f \delta^{-1}\left(A_{1}\right)=\delta f\left(A_{0}\right)$. Since $r^{-1} \delta f\left(A_{0}\right) \subset B_{u}^{0}$, $\pi\left(A_{1}\right)=r g r^{-1} \delta f \delta^{-1}\left(A_{1}\right)=r g r^{-1} \delta f\left(A_{0}\right) \subset r g\left(B_{0}^{0}\right)$.

Since $\delta\left(K_{1}\right) \subset A_{1}^{0}$, the sequence:

$$
r g\left(B_{0}\right) \supset \pi\left(A_{1}\right) \supset \pi\left(\delta\left(K_{1}\right)\right) \supset \ldots \supset \pi\left(\delta\left(K_{i}\right)\right) \supset \ldots
$$

is null for $\pi \delta\left(x_{0}\right)$. Also the sequence $\left\{\delta\left(K_{i}\right)\right\}_{i=2}^{\infty}$ is null for $\delta\left(x_{0}\right)$. Finally we have $\delta\left(K_{2}\right) \cup r g\left(B_{0}\right) \subset r g\left({ }^{*} B_{0}^{0}\right) \subset D_{1}^{0}-D_{2}$.

Hence by Lemma 1 there exists $\alpha_{3} \in G_{\mathscr{K}}$, supported on $r g\left({ }^{*} B_{0}\right)$, such that $\alpha_{3} r g\left(B_{0}\right)$ $=\delta\left(K_{2}\right), \alpha_{3} \pi\left(A_{1}\right)=\delta\left(K_{3}\right)$, and $\alpha_{3} \pi\left(\delta\left(K_{i}\right)\right)=\delta\left(K_{i+3}\right), i \geqq 1$.

$\alpha_{3} r$ is a topological dilation carried by $\mathscr{B}$.

We shall now show that $d=\alpha_{3} r g r^{-1} \alpha_{3}^{-1} \delta f \delta^{-1}$ is a topological dilation.

Consider $d\left(A_{1}\right)$ :

Since $\delta f \delta^{-1}\left(A_{1}\right) \subset C_{1}$, and $\alpha_{3}$ is supported on $D_{1}, \alpha_{3}^{-1} \delta f \delta^{-1}\left(A_{1}\right)=\delta f \delta^{-1}\left(A_{1}\right)$. Hence $d\left(A_{1}\right)=\alpha_{3} r g r^{-1} \delta f \delta^{-1}\left(A_{1}\right)=\alpha_{3} \pi\left(A_{1}\right)=\delta\left(K_{3}\right)$. And since $\delta\left(K_{i}\right) \subset A_{1}^{0}$, $i \geqq 1, \alpha_{3}^{-1} \delta f \delta^{-1}\left(\delta\left(K_{i}\right)\right)=\delta f \delta^{-1}\left(\delta\left(K_{i}\right)\right)$. Hence $d\left(\delta\left(K_{i}\right)\right)=\alpha_{3} \pi\left(\delta\left(K_{i}\right)\right)=\delta\left(K_{i+3}\right)$, $i \geqq 1$. Therefore $\left\{d^{i}\left(A_{1}\right)\right\}_{i=0}^{\infty}$ is null.

We now consider $d^{-1}\left(C_{1}\right)=\delta f^{-1} \delta^{-1} \alpha_{3} r g^{-1} r^{-1} \alpha_{3}^{-1}\left(C_{1}\right)$ : Since $\alpha_{3}$ is the identity on $C_{1}, r g^{-1} r^{-1} \alpha_{3}^{-1}\left(C_{1}\right)=r g^{-1} r^{-1}\left(C_{1}\right)$. But $g^{-1} r^{-1}\left(C_{1}\right) \subset g\left(B_{0}\right)$, so $r g^{-1} r^{-1}\left(C_{1}\right) \subset r g\left(B_{0}\right)$. Hence $\alpha_{3} r g^{-1} r^{-1}\left(C_{1}\right) \subset \alpha_{3} r g\left(B_{0}\right)=\delta\left(K_{2}\right)$. Therefore $d^{-1}\left(C_{1}\right) \subset \delta f^{-1} \delta^{-1}\left(\delta\left(K_{2}\right)\right)=\delta f^{-1}\left(K_{2}\right) \subset C_{1}^{0}$.

Now consider $d^{-1}\left(\delta f^{-1}\left(K_{i}\right)\right), i \geqq 2$ : Since $\alpha_{3}$ is the identity on $\delta f^{-1}\left(K_{i}\right) \subset C_{1}$, $r^{-1} \alpha_{3}^{-1} \delta f^{-1}\left(K_{i}\right)=r^{-1} \delta f^{-1}\left(K_{i}\right)=E_{i}$. Since $g^{-1}\left(E_{i}\right) \subset g r^{-1} \delta f\left(K_{i-1}\right), r g^{-1}\left(E_{i}\right)$ $\subset r g r^{-1} \delta f\left(K_{i-1}\right)=r g r^{-1} \delta f \delta^{-1} \delta\left(K_{i-1}\right)=\pi\left(\delta\left(K_{i-1}\right)\right)$. Hence $d^{-1}\left(\delta f^{-1}\left(K_{i}\right)\right)$ $\subset \delta f^{-1} \delta^{-1} \alpha_{3} \pi\left(\delta\left(K_{i-1}\right)\right)=\delta f^{-1} \delta^{-1}\left(\delta\left(K_{i+2}\right)\right)=\delta f^{-1}\left(K_{i+2}\right), i \geqq 2$. 
So we see that $d^{-1}\left(C_{1}\right) \subset \delta f^{-1}\left(K_{2}\right), d^{-2}\left(C_{1}\right) \subset d^{-1}\left(\delta f^{-1}\left(K_{2}\right)\right) \subset \delta f^{-1}\left(K_{4}\right)$, etc. In general, $d^{-i}\left(C_{1}\right) \subset \delta f^{-1}\left(K_{2 i}\right), i \geqq 1$.

From this, together with the fact that $d^{-i}\left(C_{1}\right) \subset d^{-i+1}\left(C_{1}^{0}\right), i \geqq 1$, we conclude that $\left\{d^{-i}\left(C_{1}\right)\right\}_{i=0}^{\infty}$ is null for $\delta f^{-1}\left(x_{0}\right)$. If we set $h_{1}=\delta$ and $h_{2}=\alpha_{3} r$, then $d=\left(h_{2} g h_{2}^{-1}\right)\left(h_{1} f h_{1}^{-1}\right)$.

Let $d \in G_{\mathcal{X}^{*}}^{*}$ be an arbitrary but fixed topological dilation:

Corollary 1. If $f, g \in G\left(X, X^{\prime}\right)$, neither $f$ nor $g$ being the identity on $X^{\prime}$, then $d$ is the product of a conjugate of $f$ by a conjugate of $g$, the conjugating homeomorphisms being elements, of $G_{\mathcal{K}^{*}}^{*}$.

Proof. By Theorem 2 there exist $h_{1}, h_{2} \in G_{\mathscr{X}}^{*}$ such that $\bar{d}=\left(h_{2} g h_{2}^{-1}\right)\left(h_{1} f h_{1}^{-1}\right)$, where $\bar{d}$ is a topological dilation. But by Theorem 1 there exists $h_{3} \in G_{\mathscr{X}}^{*}$ such that $h_{3} d h_{3}^{-1}=d \bar{d}$. Hence $h_{3} d h_{3}^{-1}=\left(h_{2} g h_{2}^{-1}\right)\left(h_{1} f h_{1}^{-1}\right)$. Therefore

$$
d=h_{3}^{-1}\left(h_{2} g h_{2}^{-1}\right)\left(h_{1} f h_{1}^{-1}\right) h_{3}=\left(h_{3}^{-1} h_{2} g h_{2}^{-1} h_{3}\right)\left(h_{3}^{-1} h_{1} f h_{1}^{-1} h_{3}\right) .
$$

Corollary 2. If $f \in G\left(X, X^{\prime}\right)$ and either $f \mid X^{\prime} \neq e$ or $f=e$ and if $d \in G\left(X, X^{\prime}\right)$, then $f$ is a product of two conjugates of $d$, the conjugating homeomorphisms being elements of $G_{\mathscr{K}}^{*}$.

Proof. If $f . \mid X^{\prime} \neq e$, then by Corollary 1 , with $d^{-1}$ as $g$, we have

$$
d=\left(h_{2} d^{-1} h_{2}^{-1}\right)\left(h_{1} f h_{1}^{-1}\right)
$$

for some $h_{2}, h_{1} \in G_{\mathscr{X}}^{*}$. Hence $\left(h_{2} d h_{2}^{-1}\right) d=h_{1} f h_{1}^{-1}$; or $h_{1}^{-1}\left(h_{2} d h_{2}^{-1}\right) d h_{1}=f$. Therefore $\left(h_{1}^{-1} h_{2} d h_{2}^{-1} h_{1}\right)\left(h_{1}^{-1} d h_{1}\right)=f$.

If $f=e$, then notice that $d^{-1}$ is a topological dilation, and hence, for some $h_{1} \in G_{\mathscr{K}}^{*}, h_{1} d h_{1}^{-1}=d^{-1}$. Then $d\left(h_{1} d h_{1}^{-1}\right)=d d^{-1}=e=f$.

Corollary 3. Suppose $f, g, h$, and $k$ are elements of $G\left(X, X^{\prime}\right)$, none of which are the identity on $X^{\prime}$. Then $f$ is the product of a conjugate of $g$ by a conjugate of $h$ by a conjugate of $k$, the conjugating elements being in $G_{\mathscr{X}}^{*}$.

Proof. By Corollary $1\left(h_{1} f h_{1}^{-1}\right)\left(h_{2} g^{-1} h_{2}^{-1}\right)=d=\left(h_{3} k h_{3}^{-1}\right)\left(h_{4} h h_{4}^{-1}\right)$ for some $h_{1}, h_{2}, h_{3}, h_{4} \in G_{\mathscr{X}}^{*}$. Then $h_{1} f h_{1}^{-1}=\left(h_{3} k h_{3}^{-1}\right)\left(h_{4} h h_{4}^{-1}\right)\left(h_{2} g h_{2}^{-1}\right)$. Hence $f=\left(h_{1}^{-1} h_{3} k h_{3}^{-1} h_{1}\right)\left(h_{1}^{-1} h_{4} h h_{4}^{-1} h_{1}\right)\left(h_{1}^{-1} h_{2} g h_{2}^{-1} h_{1}\right)$.

Corollary 4. Suppose $f, g \in G\left(X, X^{\prime}\right)$ and $f\left|X^{\prime} \neq e \neq g\right| X^{\prime}$. Then $f$ is the product of three conjugates of $g$, the conjugating elements being in $G_{\mathscr{X}}^{*}$.

Proof. Corollary 3 with $h=k=g$.

4. Examples. We now make mention of a few examples of $A$-quadruples. In each instance, of course, the existence of topological dilations is guaranteed by the corollary to Lemma 3. However in the examples to follow a canonical topological dilation $d$, suggests itself and in some instances will be given. Then, by Theorem 
1 , the collection of topological dilations coincides with the collection of $G_{\mathcal{X}^{*}}^{*}$-conjugates of $d$.

(1) Let $X=X^{\prime}=S_{n}$, the $n$-sphere for $n \geqq 1$. Let $G\left(X, X^{\prime}\right)=G_{\mathscr{X}}^{*}=$ the group of stable $\left({ }^{4}\right)$ homeomorphisms of $S_{n}$ onto itself. $\mathscr{K}$ is the collection of images of a geometric $n$-cell under the elements of $G_{\mathscr{x}}^{*}$. That Axioms (1), (2), and (3) are satisfied in this case is evident. The proof that Axioms (4) and (5) obtain may be found in [3]. Let $p \in S_{n}$ and let $q$ be the point of $S_{n}$ antipodal to $p$. We may take as $d$ an ordinary radial expansion homeomorphism of $S_{n}$ onto itself which fixes only $p$ and $q$. (We think of $d$ as the natural extension to $S_{n}$ of $d^{\prime}: E_{n} \rightarrow E_{n}$ given by $d^{\prime}(x)=r_{0} x$ for some fixed, positive $r_{0} \neq 1$.)

(2) Take $X, X^{\prime}, \mathscr{K}, G^{*}$, and $d$ as above. Let $G\left(X, X^{\prime}\right)$ be the set of all homeomorphisms whichi arel the product of some geometric orientation-reversing involution, $h$, and an element of $G_{\mathscr{x}}^{*}$.

Since $d \notin G\left(X, X^{\prime}\right)$, Corollary 2 is not applicable here. However $d h \in G\left(X, X^{\prime}\right)$ so we may substitute for Corollary 2 the following: if $f \in G\left(X, X^{\prime}\right)$, then $f$ is the product of a conjugate of $d$ by a conjugate of $d h$, the conjugating homeomorphisms, of course, being in $G_{\mathscr{x}}^{*}\left({ }^{5}\right)$.

(3) Let $X=S_{n}, n \geqq 1$. Let $X^{\prime}$ be a countable dense subset of $X$, and let $G_{\mathscr{X}}^{*}=G\left(X, X^{\prime}\right)$ be those stable homeomorphisms which take $X^{\prime}$ onto $X^{\prime} . \mathscr{K}$ is the collection of images of a geometric $n$-cell under elements of $G^{*}{ }_{\mathscr{X}}$.

(4) Let $X=D_{n}=$ the $n$-cell, $n \geqq 2$. Call $X^{\prime}$ the boundary of $D_{n}$, and $G_{\mathscr{X}}$ is the set of all homeomorphisms of $D_{n}$ onto itself each of which is the identity on some neighborhood of some point of $X^{\prime} . G\left(X, X^{\prime}\right)=G_{\mathscr{X}}^{*}$, and $\mathscr{K}$ is the collection of images of a geometric half-cell under the elements of $G_{\mathscr{X}}^{*}$. Axioms (1), (2), and (3) are obviously satisfied, and Axioms (4) and (5) follow from arguments like those given in [3].

(5) Let $X=X^{\prime}$ be the rationals, the irrationals, or the Cantor set. Let $G_{\mathscr{X}}^{*}=G\left(X, X^{\prime}\right)$ be the collection of all homeomorphisms of $X$ onto itself. $\mathscr{K}$ is the collection of all nonvoid, proper, open and closed subsets of $X$.

If $X$ is the Cantor set, realized as the 'middle third' set in $[0,1]$, define $\left\{C_{i}\right\}_{i=-\infty}^{\infty}$ as follows: for $i \leqq 0$,

$C_{i}=\left[\frac{2}{3^{-i+2}}, \frac{1}{3^{-i+1}}\right] \cap X$, and for $i \geqq 1, C_{i}=\left[\frac{3^{i}-1}{3^{i}}, \frac{3^{i+1}-2}{3^{i+1}}\right] \cap X$.

Then $\bigcup_{i=-\infty}^{\infty}\left\{C_{i}\right\} \cup\{0\} \cup\{1\}=X$. Take $d$ as any homeomorphism of $X$ onto itself such that $d(0)=0, d(1)=1$, and $d\left(C_{i}\right)=C_{i+1},-\infty<i<\infty$.

(4) $G_{\mathscr{X}}^{*}$ is the group generated by the collection of all those homeomorphisms of $S_{n}$ onto itself each of which is the identity on some nonvoid open set. The term 'stable' used to denote this group appears to be due to Brown and Gluck [3].

(5) If the annulus problem is solved affirmatively, then in (1) we may take $G_{\mathscr{K}}^{*}=G\left(X, X^{\prime}\right)$ to be the collection of all orientation-preserving homeomorphisms, and in (2) we may take $G\left(X,{ }^{\prime} X\right)$ to be all the orientation-reversing homeomorphisms. 
A few words about possible extensions of the preceding conclusions. Let $G$ denote the group of stable homeomorphisms of $E_{n}$. Set $d(x)=r_{0} x, x \in E_{n}, r_{0}>1$. By arguments similiar in spirit to those of Theorems 1 and 2, one can show that if $f \in G_{n}$, then there exist stable homeomorphisms $\delta$ and $r$ such that $\delta d \delta^{-1} f=r d r^{-1}$. Therefore $f$ is the product of a stable conjugate of $d$ by a stable conjugate of $d^{-1}$.

It is impossible to obtain Corollary 4 for the group $G_{n}$, since it is not simple. However the following question might well be asked: suppose $f, g \in G_{n}$ with $f$ not the identity and $g$ not supported on a cell. Is $f$ the product of three conjugates of $g$ ? Or if three won't work, what is the smallest number which will? Three appears to suffice, if $g$ moves a tame ray off of itself.

Finally we remark that the number of conjugates of $g$ employed in Corollary 4 cannot, in general, be reduced: In each of the examples (1)-(5) above it is easy to find nonidentity elements $f$ and $g$ of $G\left(X, X^{\prime}\right)$ such that $f$ is not the product of two conjugates of $g$.

\section{BIBLIOGRAPHY}

1. R. D. Anderson, The three conjugates theorem, Topology (to appear).

2. - The algebraic simplicity of certain groups of homeomorphisms, Amer. J. Math., 80 (1958), 955-963.

3. Morton Brown and Herman Gluck, Stable structures on manifolds. I, Ann. of Math. 79 (1964), 1-17.

4. Gordon Fisher, On the group of all homeomorphisms of a manifold, Trans. Amer. Math. Soc. 97 (1960), 193-212.

5. J. M. Kister, Isotopies in 3-manifolds, Trans. Amer. Math. Soc. 97 (1960), 213-224.

6. H. Kneser, Die Deformationssatz der Einfach Zusammenhangenden Flachen, Math. Z. 25 (1926), 362-372.

7. S. Ulam and J. von Neumann, On the group of homeomorphisms of the surface of a sphere, Abstract 283, Bull. Amer. Math. Soc. 53 (1947), 506.

8. N. J. Fine and G. E. Schweigert, On the group of homeomorphisms of an arc, Ann. of Math. 62 (1955), 237-253.

9. M. E. Hamstrom and E. Dyer, Regular mappings and the space of homeomorphisms on a 2-manifold, Duke Math. J. 25 (1958), 521-531.

10. M. E. Hamstrom, Regular mappings and the space of homeomorphisms on a 3-manifold, Abstract 564-39, Notices Amer. Math. Soc. 6 (1959), 783-784.

11. J. H. Roberts, Local arcwise connectivity in the space $H_{n}$ 'of homeomorphisms of $S^{n}$ onto itself, Summary of Lectures, Summer Institute on Set Theoretic Topology, Madison, Wisconsin, 1955; p. 100.

12. M. K. Fort, Jr., A proof that the group of homeomorphisms of the plane onto itself is locally arcwise connected, Proc. Amer. Math. Soc. 1 (1950), 59-62.

Louisiana State University,

Baton Rouge, Louisiana 